\title{
Identification of serum miRNAs differentially expressed in human epilepsy at seizure onset and post-seizure
}

\author{
JIJUN SUN ${ }^{1}$, WEIDONG CHENG ${ }^{1}$, LIFENG LIU ${ }^{1}$, SHUXIN TAO $^{1}$, \\ ZHANGYONG XIA ${ }^{1}$, LIFENG QI ${ }^{1}$ and MIN HUANG ${ }^{2}$ \\ ${ }^{1}$ Department of Neurology, Liaocheng People's Hospital and Liaocheng Clinical School of Taishan Medical University, \\ Liaocheng, Shandong 252000; ${ }^{2}$ Department of Neurology, Second Clinical College, \\ Jinan University, Shenzhen, Guangdong 518020, P.R. China
}

Received September 14, 2015; Accepted September 22, 2016

DOI: $10.3892 / \mathrm{mmr} .2016 .5906$

\begin{abstract}
MicroRNAs (miRNAs) function as potential novel biomarkers for disease detection due to their marked stability in the blood and the characteristics of their expression profile in several diseases. In the present study, microarray-based serum miRNA profiling was performed on serum obtained from three patients with epilepsy at diagnosis and from three healthy individuals as controls. This was followed by reverse transcription-quantitative polymerase chain reaction analysis in a separate cohort of 35 health volunteers and 90 patients with epilepsy. The correlations between miRNAs and clinical parameters were analyzed. The array results showed that 15 miRNAs were overexpressed and 10 miRNAs were underexpressed (>2-fold) in the patients with epilepsy. In addition, four miRNAs, including miR-30a, miR-378, miR-106b and miR-15a were found to be overexpressed in the serum of patients at seizure onset, compared with post-seizure. When the patients were at seizure onset, the expression of miR-30a was positively associated with seizure frequency. No significant differences were found between miR-30a and gender, age or number of years following diagnosis. The expression levels of miR-378, miR-106b and mir-15a were not associated with the clinical parameters in the patients with seizures. Calcium/calmodulin-dependent protein kinase type IV was a target of miR-30a, and its expression was increased following seizure and was negatively correlated with miR-30a in the patients with epilepsy. The present study provided the first evidence, to the best of our knowledge, that the expression levels of miR-378, miR-30a, miR-106b and miR-15a were enhanced in epileptic patients with seizures. miR-30a may be useful for prognostic prediction in epilepsy.
\end{abstract}

Correspondence to: Dr Min Huang, Department of Neurology, Second Clinical College, Jinan University, 1017 North Dongmen Road, Shenzhen, Guangdong 518020, P.R. China

E-mail: huang_min_jnu@163.com

Key words: epilepsy, seizure, microRNA-30a, prognosis

\section{Introduction}

Epilepsy is a common chronic neurological disease, which is characterized by recurring seizures. It has been reported that the morbidity rate of epilepsy is $\sim 1 \%$ worldwide (1). Epilepsy affects $>65,000,000$ individuals in the world and $>100,000$ new cases are diagnosed every year (2). Electroencephalography (EEG) and magnetic resonance imaging (MRI) are the most widely used techniques for the diagnosis of epilepsy. EEG and MRI can detect and locate the epileptic seizures and zones (3). Usually, a typical EEG of epilepsy ranges between minutes and hours (4). However the exact etiology of epilepsy remains to be fully elucidated.

MicroRNAs (miRNAs) are small non-coding RNAs (ncRNAs) and are the most well-researched family of ncRNAs to date. Mature miRNA sequences are 22 nucleotides in length (5). They are involved in depression of the expression of post-transcriptional target genes via binding to the 3'-untranslated region (3'UTR) (6). miRNAs are abundant in the nervous system where they serve as effectors of brain development and in maintenance of the neuronal phenotype (7).

The results of previous expression profiling have shown unique miRNA expression in acute brain injuries (8). Several studies have demonstrated that miRNAs affect the pathomechanisms in epileptogenic injuries (9). Previously, four studies have profiled the expression of miRNAs in experimental epilepsy, in which $>100$ different miRNAs were identified. These results confirmed that epilepsy is associated with widespread alterations in the expression of miRNAs (10-13). The expressed miRNAs were all detected in the brain tissues of patients or animal models. The present study aimed to examine the serum expression of miRNAs in patients with epilepsy, and to select differently expressed miRNA between seizure onset and post-seizure to explore the function of selected miRNA in epilepsy. This was performed with the aim to identify the biomarker of epilepsy treatment.

\section{Materials and methods}

Patients and serum samples. Following ethical approval and the provision of written informed consent, serum samples were collected from three seizure patients for miRNA microarray 
analysis and from another 90 patients for confirmation of the miRNA expression. The patients were evaluated by an epileptologist and underwent a basal EEG and 3T MR brain imaging. Patients experiencing symptoms and EEG semiology were considered to be at seizure onset. Following treatment, when the symptoms and EEG semiology tended to be normal, patients were considered to be post-seizure. All samples were collected from consenting individuals from the Liaocheng People's Hospital, Taishan Medical College (Liaocheng, China) and Jinan University (Shenzhen, China). The sample collection was performed according to the protocols approved by the Ethics Committee of Jinan University. The blood samples were collected at seizure onset and post-seizure.

RNA isolation from human serum samples. Venous blood $(5 \mathrm{ml})$ was collected and stored at room temperature for $1 \mathrm{~h}$, followed by centrifugation at $1,600 \times \mathrm{g}$ for $10 \mathrm{~min}$ at $4^{\circ} \mathrm{C}$. The serum was gently collected and stored at $-80^{\circ} \mathrm{C}$ or used in the following experiment. For all experiments, $500 \mu \mathrm{l}$ of human serum was used and total RNA was extracted using TRIzol reagent (Thermo Fisher Scientific, Inc., Waltham, MA, USA) The concentration and the 260/280 $\mathrm{nm}$ absorbance ratio was detected using a Nanodrop 2000 (Thermo Fisher Scientific, Inc., Pittsburgh, PA, USA).

MicroRNA expression profiling. Total RNA (100 ng) was used to synthesize first strand cDNA at $42^{\circ} \mathrm{C}$ for $2 \mathrm{~h}$ using ArrayScript ${ }^{\mathrm{TM}}$ reverse transcriptase, second strand cDNA was at $16^{\circ} \mathrm{C}$ for $1 \mathrm{~h}, 65^{\circ} \mathrm{C}$ for $10 \mathrm{~min}$ using reverse transcriptase and DNA polymerase, respectively. T7 Biotin IVT Mix was used to generate multiple copies of biotin-modified aRNA from the double stranded cDNA templates, incubated for 2-14 h at $40^{\circ} \mathrm{C}$ (MessageAmp ${ }^{\mathrm{TM}}$ Premier RNA Amplification kit; Thermo Fisher Scientific, Inc.). miRNAs were individually detected using specific oligonucleotides (Takara Bio, Inc.). A single miRNA-specific oligonucleotide was designed against each mature miRNA sequence, and miRNA-specific primers were extended using DNA polymerase. Universal primers were used to amplify the cDNA templates and the primer complimentary to the array was fluorescently labeled using miRCURY Hy3/Hy5 Power Labeling kit (Takara Bio Inc.). The labeled, single-stranded PCR products were hybridized to a Human v2.0 miRNA Expression BeadChip (Illumina, Inc., San Diego, CA) with 1,146 human miRNAs (97\% coverage in the miRBase 12.0 database) $(14,15)$.

Reverse transcription-quantitative PCR (RT-qPCR) analysis of the expression of miRNAs and mRNAs. RT for the individual qPCR analyses was performed using $500 \mathrm{ng}$ of total RNA and a Reverse Transcription kit (Promega Corporation, Madison, WI, USA). RT-specific primers for human miRNAs miR-378, miR-30a, miR-106b and miR-15a (Applied Biosystems; Thermo Fisher Scientific, Inc.) were used for all miRNA RT procedures. Individual qPCR analyses were performed on the 7900HT Fast Realtime system (Applied Biosystems; Thermo Fisher Scientific, Inc.) using miR-378-, miR-30a-, miR-106b- and miR-15a-specific Taqman miRNA assays (Applied Biosystems Thermo Fisher Scientific, Inc.). RNU6B was used for the normalization of miRNA expression levels. The mRNA levels of calcium/calmodulin-dependent protein kinase type IV (CAMK4), were determined using TaqMan Real-Time PCR analysis, according to manufacturer's protocols (Applied Biosystems; Thermo Fisher Scientific, Inc.). To determine mRNA expression levels, total RNA (10 ng) were reverse transcribed using iScript Reverse Transcription Supermix (Bio-Rad Laboratories, Inc., Hercules, CA, USA). The cDNA templates were amplified with TaqMan PreAmp Master mix (Applied Biosystems; Thermo Fisher Scientific, Inc.). The PCR programs consisted in a hot start of $95^{\circ} \mathrm{C}$ for $10 \mathrm{~min}$, followed by 40 cycles of $15 \mathrm{sec}$ at $95^{\circ} \mathrm{C}$ and $1 \mathrm{~min}$ at $60^{\circ} \mathrm{C}$. The primers used were: CAMK4, sense $5^{\prime}-\mathrm{AGC}$ ACATTCAAACCACCACA-3', antisense 5'-GGACTCAGA GATCCGTCTGC-3'; miR-30a, sense 5'-TGTAAACAT CCTCGAC-3', antisense 5'-ACATCCAGTGTAGCATA-3'; miR-378, sense 5'-ACACTCCAGCTGGGACTGGACTTG GAGTC-3', antisense 5'-TGGTGTCGGAGTCG-3'; miR-106a, sence 5'-CGGAATTCATCTCGAGACGCCAACTTG-3', antisense 5'-CGGGATCCCTTCATTCAAGGTCAATGAG-3'; miR-15a, sense 5'- GTCGTATCCAGTGCAGGGTCCGAG GTATTC-3', antisense 5'-GCACTGGATACGACCACA AA-3'; RNU6B, sense 5'-CTCGCTTCGGCAGCACA-3', antisense 5'-AACGCTTCACGAATTTGCGT-3'; and GAPDH, 5'-AAGGTCGGAGTCAACGGATT-3', antisense 5'-CTGGA AGATGGTGATGGGATT-3'. Each sample was analyzed in triplicate, in three independent experiments. The level of each mRNA was measured using the quantification cycle $(\mathrm{Cq})$ and the level of the target was calculated as described above for the miRNAs. The gene expression levels were normalized against the expression of GAPDH. The relative fold change in expression of the target gene transcript was determined using the comparative quantification method $\left(2^{-\Delta \Delta \mathrm{Cq}}\right)(16)$. High or low expression of miRNAs and mRNAs were defined on the basis of the median expression, determined separately for each cohort.

Construction of luc-UTR vectors. The full-length CAMK4 3'-UTRs were cloned into the EcoRI and HindIII sites of the pMIR-REPORT luciferase vector (Ambion; Thermo Fisher Scientific, Inc.) using a PCR-generated fragment. A Luc-mut vector, in which the first seven nucleotides complementary to the miR-30a seed-region were mutated, served as a mutant control. The binding sites of CAMK4 were UGUUUACA and these were replaced with the CACCCGUG in the Luc-mut vector.

Luciferase reporter assay. The Luc-wild-type (wt), Luc-mut and Luc-control vectors were co-transfected within miR-30a mimics into 293T cells (Cell bank of Chinese Academy of Sciences, Shanghai, China) using Lipofectamine 2000 (Thermo Fisher Scientific, Inc.). The pMIR-REPORT $\beta$-galactosidase control vector (Thermo Fisher Scientific, Inc.) was transfected into cells to serve as a control. Luciferase activity was measured in the cell lysates $48 \mathrm{~h}$ following transfection using a dual-light luminescent reporter gene assay kit (Applied Biosystems; Thermo Fisher Scientific, Inc.). The results were normalized against the activity of $\beta$-galactosidase (17).

Western blot analysis. Cells was lysed in ice-cold lysis buffer (1\% NP-40; 50 mM Tris-HCl, pH 8.0; 100 mM sodium fluoride; $30 \mathrm{mM}$ sodium pyrophosphate; $2 \mathrm{mM}$ sodium 
Table I. Association between expression level of miRNAs and clinical parameters of epilepsy.

\begin{tabular}{|c|c|c|c|c|c|c|c|c|}
\hline \multirow[b]{2}{*}{ Parameter } & \multicolumn{2}{|c|}{ miR-30a } & \multicolumn{2}{|c|}{ miR-378 } & \multicolumn{2}{|c|}{ miR-106b } & \multicolumn{2}{|c|}{ miR-15a } \\
\hline & High & Low & High & Low & High & Low & High & Low \\
\hline \multicolumn{9}{|l|}{ Gender } \\
\hline Male (52) & 36 & 16 & 28 & 24 & 27 & 25 & 37 & 15 \\
\hline Female (38) & 25 & 13 & 26 & 12 & 21 & 17 & 20 & 18 \\
\hline \multicolumn{9}{|l|}{ Age } \\
\hline$\geq 39$ years $(41)$ & 28 & 13 & 26 & 15 & 25 & 16 & 26 & 15 \\
\hline <39 years (49) & 33 & 16 & 28 & 21 & 23 & 26 & 31 & 18 \\
\hline \multicolumn{9}{|l|}{ Years diagnosed } \\
\hline$\geq 16(32)$ & 20 & 11 & 17 & 15 & 18 & 14 & 19 & 13 \\
\hline$<16(58)$ & 41 & 18 & 37 & 21 & 30 & 28 & 38 & 20 \\
\hline \multicolumn{9}{|l|}{ Seizure frequency } \\
\hline$\geq 5 /$ month (37) & 31 & $6^{\mathrm{a}}$ & 22 & 14 & 21 & 15 & 25 & 12 \\
\hline$<5 /$ month (53) & 30 & 23 & 32 & 21 & 26 & 27 & 32 & 21 \\
\hline
\end{tabular}

High and low expression were defined on the basis of the median expression. ${ }^{a} \mathrm{P}<0.01$, compared with high expression. miR/miRNA, microRNA

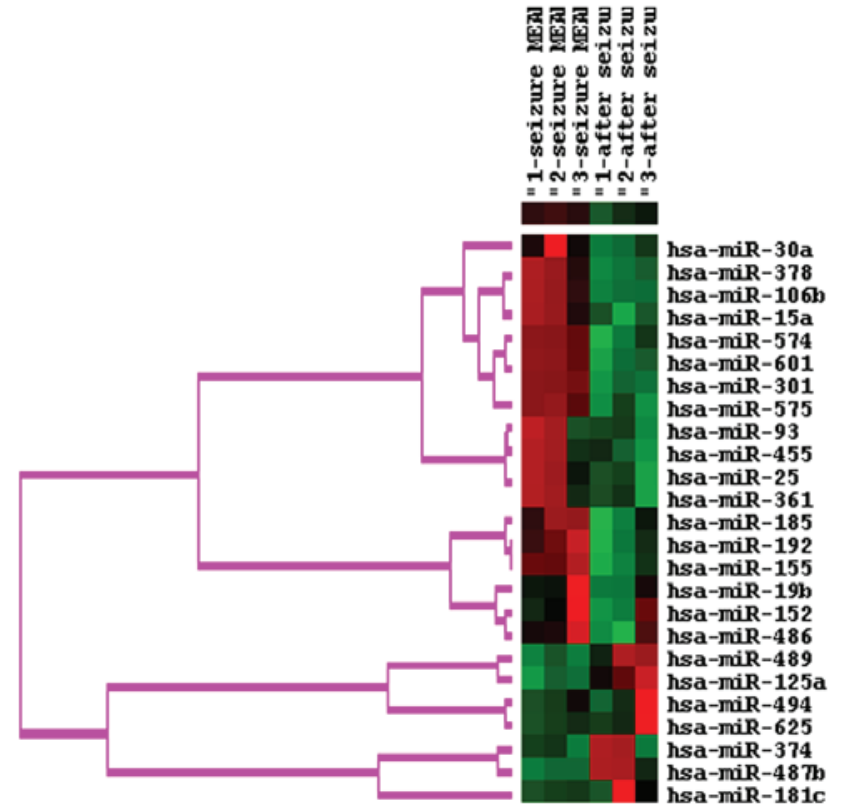

Figure 1. miRNAs differentially expressed in patients with epilepsy at seizure onset and post-seizure. Heat map representation of the average expression levels of the 25 miRNAs differentially expressed at seizure onset and post-seizure in serum from three patients. The colors of the genes represented on the heat map correspond to the expression values normalized againsy the mean expression of miRNA across all samples. Green indicates downregulated expression and red indicates upregulated expression in the serum. miRNA/miR, microRNA.

molybdate; $5 \mathrm{mM}$ EDTA and $2 \mathrm{mM}$ sodium orthovanadate). Lysates were centrifuged at $10,000 \mathrm{x} g$ for $15 \mathrm{~min}$ at $4^{\circ} \mathrm{C}$. The concentration was determined by Pierce bicinchoninic acid Protein Assay kit (Thermo Fisher Scientific, Inc.). Protein lysates $(50 \mu \mathrm{g})$ were separated on $10 \%$ SDS-polyacrylamide gels and electrophoretically transferred onto polyvinylidene difluoride membranes (EMD Millipore, Billerica, MA, USA).
Membranes were blocked in 5\% nonfat milk in TBS-Tween (TBST) for $1 \mathrm{~h}$ at room temperature. The primary rabbit anti-human CaMK4 antibody (cat. no. 4032; Cell Signaling Technology, Inc., Danvers, MA, USA) was diluted 1:1,000 in $5 \%$ milk with TBST and incubated with the membrane at $4^{\circ} \mathrm{C}$ overnight. Then blots were washed three times with TBST. Secondary goat anti-rabbit IgG (cat. no. ab6721; Abcam, Cambridge, UK) was diluted at 1:3,000 in milk with TBST and incubated with the membrane at $37^{\circ} \mathrm{C}$ for $1 \mathrm{~h}$. The hybridization signals were detected by chemiluminescence (Invitrogen; Thermo Fisher Scientific, Inc.) and captured using a ChemiDOC (Bio-Rad Laboratories, Inc.).

Statistical analysis. All data are presented as the mean \pm standard error of the mean. Analysis was performed using SPSS software (version 17; SPSS, Inc., Chicago, IL, USA).Comparisons between two groups were made using Student's $t$-test, whereas multigroup comparisons were made using analysis of variance followed by appropriate post-hoc testing. Pearson's correlation analysis was used to test the correlation between two groups. $\mathrm{P}<0.05$ was considered to indicate a statistically significant difference.

\section{Results}

Clinical parameters. Serum from three patients at seizure onset and post-seizure were used for microarray analysis. The patients included two men and one woman. Serum from an additional 90 patients at seizure onset and post-seizure were used for RT-qPCR analysis. The patients had a mean age of 39 years (range, 21-60), a mean number of years following epilepsy diagnosis of 16 years (range, 5-31) and a mean seizure frequency of 5 (range, 1-20) times per month.

miRNA microarray. The expression levels of 15 miRNAs were increased and 10 miRNAs were decreased at seizure onset, 
A

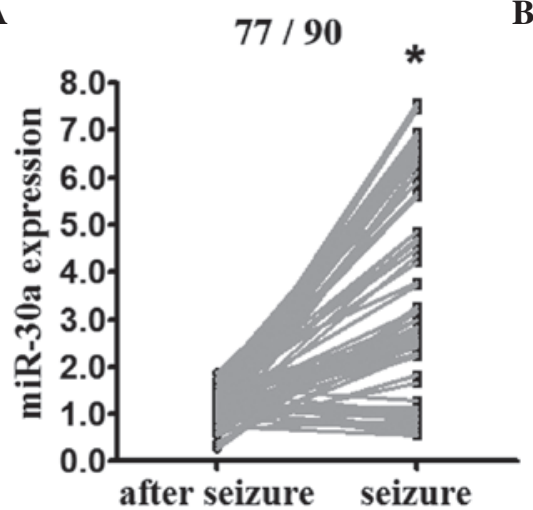

C

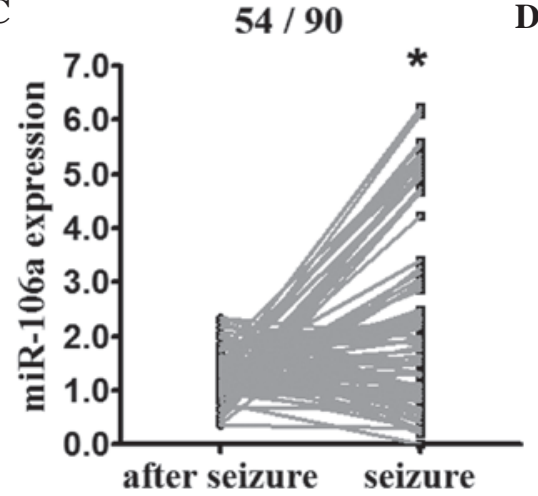

D
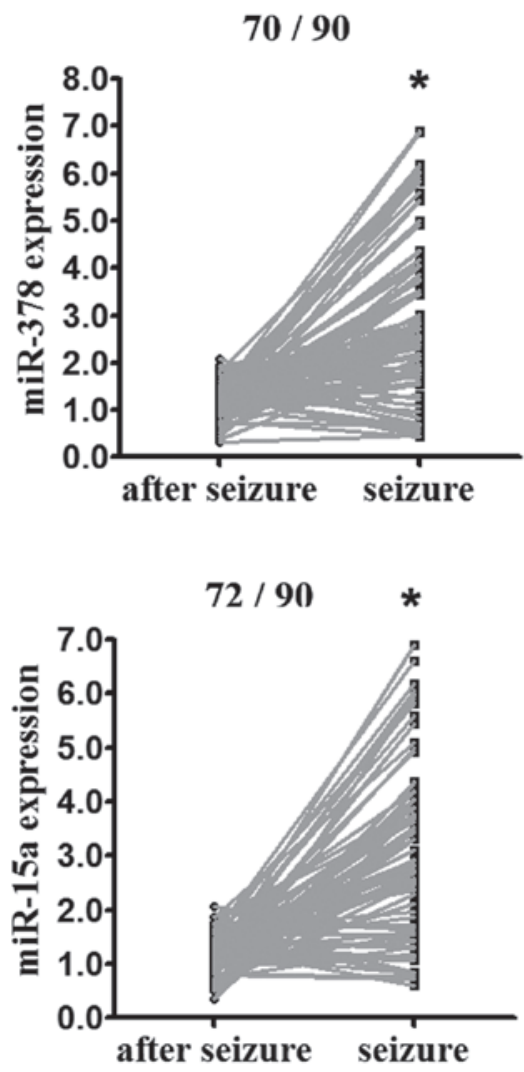

Figure 2. Relative expression of (A) miR-30a, (B) miR-378, (C) miR-106b and (D) miR-15a, evaluated using reverse transcription-quantitative polymerase chain reaction analysis, in the additional cohort of patients with epilepsy (90 patients per group). Values (above graphs) indicate the number of samples that the miR was decreased in after seizure out of the total number of samples examined. The expression levels of miR-30a, miR-378, miR-106b and miR-15a were detected at seizure onset seizures and post-seizure. ${ }^{*} \mathrm{P}<0.01$. miR, microRNA.

compared with post-seizure in the patients with epilepsy, as determined using miRNA microarray analysis (Fig. 1). The expression of miR-30a, miR-378, miR-106b and miR-15a was confirmed in the extended cohort of the three original patients and the additional 90 patients (indicated in Table I), using RT-qPCR analysis. The expression levels of miR-30a, miR-378, miR-106b and miR-15a, the top four overexpressed miRNAs identified, were enhanced at seizure onset, compared with post-seizure (Fig. 2).

Correlation between upregulated miRNAs and clinical parameters in patients experiencing seizure. The correlation between upregulated miRNAs and clinical parameters was statistically analyzed. The expression levels of miR-378, miR-106b and miR-15a were not associated with the age, gender, years following diagnosis or seizure frequency. The expression of miR-30a was positively associated with seizure frequency (Table I).

CAMK4 is a target of $m i R-30 a$. The online software program, Targetscan 6.0 (www.targetscan.org/vert_71/), was used to assist in identifying miR-30a targets. The miR-30a mimics decreased the luciferase activity of the CAMK4-3'-UTR-wt reporter (Fig. 3A). The results from the RT-qPCR and western blot analyses showed that the enhanced expression of miR-30a by the miR-30a mimics in the $293 \mathrm{~T}$ cells led to a decrease in endogenous mRNA and protein levels of CaMK4 (Fig. 3B). The mRNA expression of CAMK4 was significantly reduced post-seizure, compared with at seizure onset (64/90; Fig. 3C). The expression of miR-30a and CAMK4 were demonstrated to be negatively correlated (Fig. 3D).

\section{Discussion}

The pathogenesis of different types of epilepsy involves several important biological pathways, a number of which have been shown to be regulated by miRNAs. In the present study, the expression of miRNAs was compared at seizure onset with expression post-seizure in patients with epilepsy. The miRNA microarray revealed that the expression levels of 15 miRNAs were increased and 10 miRNAs were decreased at seizure onset, compared with at post-seizure in patients with epilepsy. The present study then confirmed that the expression levels of miR-30a, miR-378, miR-106b and miR-15a were higher at seizure onset, compared with levels post-seizure in the serum of patients with epilepsy.

miR-30a is involved in tumorigenesis, inflammation and myoblast differentiation and miR-30a is known to function as a tumor suppressor in breast cancer, small cell lung cancer and colorectal carcinoma (18). miR-30a has been found to be increased in cerebral arteries following subarachnoid hemorrhage (SAH), compared with the levels in rats subjected to sham surgery, and may be involved in the vascular wall changes observed following SAH (19). Wen et al (20) demonstrated that miR-30a-5p is significantly overexpressed in hepatitis B virus (HBV)-positive patients 
A

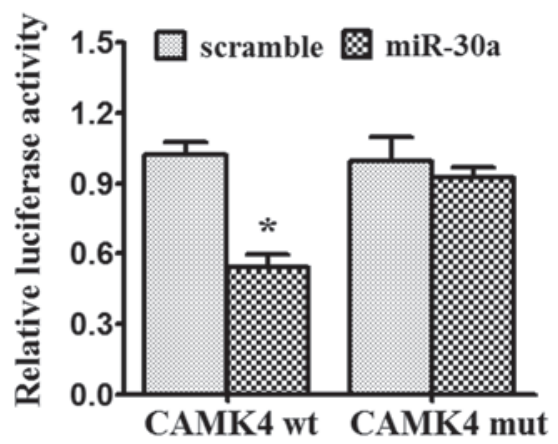

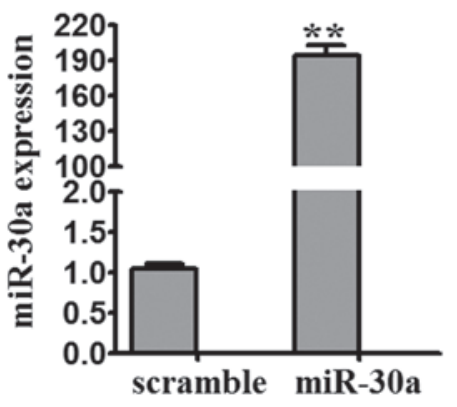

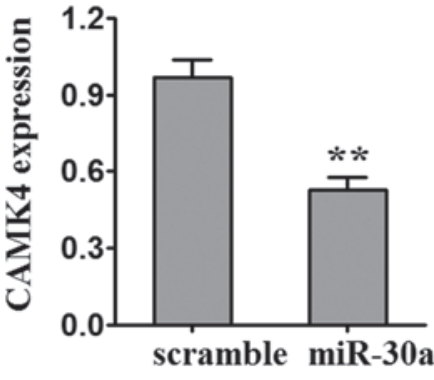

CAMK4

$\beta$-actin
C

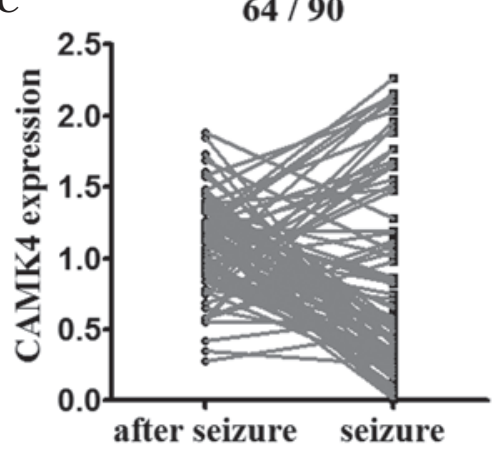

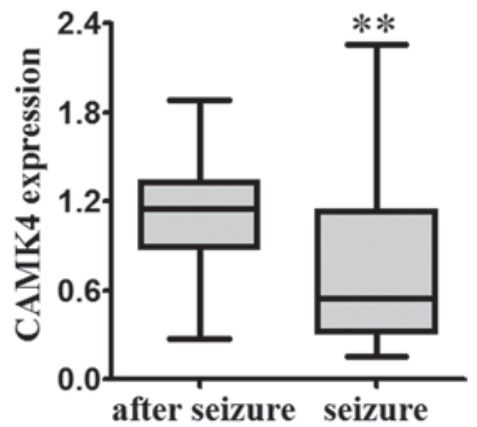

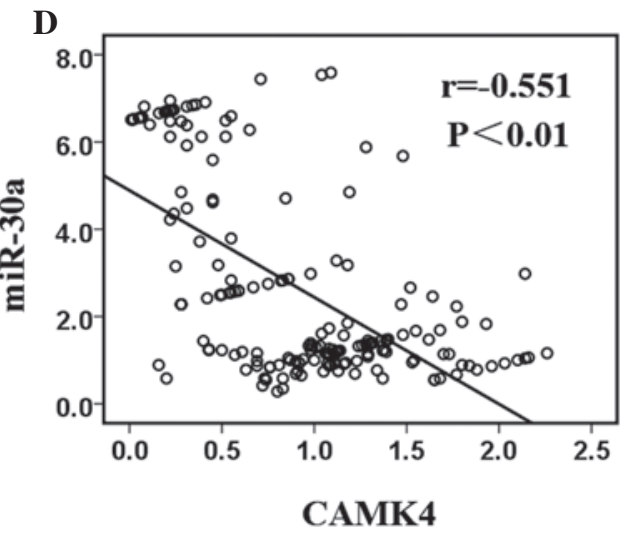

Figure 3. CAMK4 is a target of miR-30a in epilepsy. (A) miR-30a was found to bind to the CAMK4 3' untranslated region, as determined using a luciferase reporter assay. (B) Expression of CAMK4 was decreased by miRNA-30a, determined using reverse transcription-quantitative polymerase chain reaction and western blot analyses. ${ }^{*} \mathrm{P}<0.05,{ }^{* *} \mathrm{P}<0.01$ vs. scramble. (C) Expression of CAMK4 in patients with epilepsy. CAMK4 expression was decreased in $64 / 90$ samples after seizure compared with at seizure onset. (D) Correlation between miR-30a and CAMK4 in epilepsy. ${ }^{* *} \mathrm{P}<0.01$ vs. after seizure. miR, microRNA; CAMK4, calcium/calmodulin-dependent protein kinase type IV.

with hepatocellular cancer, compared with HBV-positive controls without cancer. This study revealed that plasma miR-30a offers potential as an early biomarker for detecting hepatocellular carcinoma. Overexpression of miR-30a-5p promotes the differentiation of myoblasts, whereas its inhibition restricts the differentiation of myoblasts in vitro (21). Circulating levels of miR-30a have been found to be markedly downregulated in patients with ischemic stroke until 24 weeks (22). As all four miRNAs detected in the present study were decreased following seizures, the present study analyzed the association between the expression of miRNAs in patients with epilepsy with seizures and the clinical parameters. The results of the present study revealed that the expression of miR-30a was higher in the sera of patients at seizure onset, with that post-seizure, and was associated with seizures frequency. Several studies have indicated that miR-30a functions in multiple biological processes via targeting a number of genes. For example, miR-30a can target insulin receptor substrate 2 in colorectal tumorigenesis (23). The overexpression of miR-30a upregulates the expression levels of B cell lymphoma 2-related protein A1 immediate early response 3 and cyclin D2 by inhibiting forkhead transcription factor ligand 2 (24). The downregulation of miRNA-30a alleviates cerebral ischemic injury through enhancing beclin 1-mediated autophagy (25). In the present study, the hypothesis that CAMK4 was a target of miR-30a was confirmed. The inhibition of CAMK4 is detrimental in cerebral ischemia (26). The present study analyzed the correlation between the expression levels of miR-30a and CAMK4. The expression of CAMK4 was negatively associated with that of miR-30a in patients with epilepsy. However, the function of CAMK4 in epilepsy was not investigated, and CAMK4 may be involved in the miR-30a-mediated pathway in epilepsy.

miR-378 promotes the migration of liver cancer cells by downregulating the expression of Fus (27). miR-378 is also considered to be a diagnostic biomarker in cancer, including renal cell carcinoma (28) and colorectal cancer (29). miR-378 may be a potential biomarker for characterizing non-small cell lung cancer brain metastasis (30). The overexpression of miR-378 attenuates high glucose-suppressed osteogenic differentiation through targeting caspase 3 and activating the phosphoinositide 3 -kinase/Akt signaling pathway (31). Circulating levels of miR-378 predicts left ventricular hypertrophy in patients with aortic stenosis (32). miRNA-378 controls classical brown fat expansion to counteract obesity (33). miR-106b promotes hematopoietic cell expansion by targeting sequestosome 1-regulated pathways in mice (34). miR-106b is decreased in patients with chronic myeloid leukemia in the chronic phase (35). 
A previous study found that the relative levels of miR-106b prior to and following Helicobacter pylori eradication were significantly higher in the high-risk group, compared with the control (36). miR-15a enhances the radiosensitivity of breast cancer cells by targeting G2 checkpoints (37). Maudet et al (38) identified the miR-15a miRNA family as cellular restriction factors for Salmonella infection using functional high-throughput screening. The miR-15 family is a regulator of cardiac hypertrophy and fibrosis, acting by inhibiting of the transforming growth factor $\beta$-pathway (39). However, the functions of miR-378, miR-106b and miR-15a in the nervous system have not been reported. In the present study, it was found that miR-378, miR-106b and miR-15a were increased in the sera of patients experiencing seizures. The mechanisms underlying the regulation of the expression of these miRNAs require further investigation. The present study suggested that miR-30a may be useful for predicting prognosis following seizure.

\section{References}

1. van Graan LA, Lemieux L and Chaudhary UJ: Methods and utility of EEG-fMRI in epilepsy. Quant Imaging Med Surg 5: 300-312, 2015.

2. Kwan P, Schachter SC and Brodie MJ: Drug-resistant epilepsy. N Engl J Med 365: 919-926, 2011.

3. Ahmad MA, Ayaz Y, Jamil M, Omer Gillani S, Rasheed MB, Imran M,Khan NA, Majeed W and Javaid N: Comparative analysis of classifiers for developing an adaptive computer-assisted EEG analysis system for diagnosing epilepsy. Biomed Res Int 2015: 638036, 2015.

4. Puttachary S, Sharma S, Stark S and Thippeswamy T: Seizure-induced oxidative stress in temporal lobe epilepsy. Biomed Res Int 2015: 745613, 2015.

5. Sano T, Reynolds JP, Jimenez-Mateos EM, Matsushima S, Taki W and Henshall DC: MicroRNA-34a upregulation during seizure-induced neuronal death. Cell Death Dis 3: e287, 2012.

6. Ha M and Kim VN: Regulation of microRNA biogenesis. Nat Rev Mol Cell Biol 15: 509-524, 2014.

7. Saugstad JA: Non-Coding RNAs in stroke and neuroprotection. Front Neurol 6: 50,2015.

8. Bhalala OG, Srikanth M and Kessler JA: The emerging roles of microRNAs in CNS injuries. Nat Rev Neurol 9: 328-339, 2013.

9. Liu DZ, Tian Y, Ander BP, Xu H, Stamova BS, Zhan X, Turner RJ, Jickling $G$ and Sharp FR: Brain and blood microRNA expression profiling of ischemic stroke, intracerebral hemorrhage, and kainate seizures. J Cereb Blood Flow Metab 30: 92-101, 2010.

10. Henshall DC: MicroRNA and epilepsy: Profiling, functions and potential clinical applications. Curr Opin Neurol 27: 199-205, 2014.

11. Zucchini S, Marucci G, Paradiso B, Lanza G, Roncon P, Cifelli P, Ferracin M, Giulioni M, Michelucci R, Rubboli G and Simonato M: Identification of miRNAs differentially expressed in human epilepsy with or without granule cell pathology. PLoS One 9: e105521, 2014

12. Li MM, Jiang T, Sun Z, Zhang Q, Tan CC, Yu JT and Tan L: Genome-wide microRNA expression profiles in hippocampus of rats with chronic temporal lobe epilepsy. Sci Rep 4: 4734, 2014.

13. McKiernan RC, Jimenez-Mateos EM, Sano T, Bray I, Stallings RL, Simon RP and Henshall DC: Expression profiling the microRNA response to epileptic preconditioning identifies miR-184 as a modulator of seizure-induced neuronal death. Exp Neurol 2: 346-354, 2013.

14. Zeng X, Xiang J, Wu M, Xiong W, Tang H, Deng M, Li X, Liao Q, Su B, Luo Z, et al: Circulating miR-17, miR-20a, miR-29c, and miR-223 combined as non-invasive biomarkers in nasopharyngeal carcinoma. PLoS One 7: e46367, 2012.

15. You G, Yan W, Zhang W, Wang Y, Bao Z, Li S, Li S, Li G, Song Y, Kang C and Jiang T: Significance of miR-196b in tumor-related epilepsy of patients with gliomas. PLoS One 7: e46218, 2012.
16. Livak KJ and Schmittgen TD: Analysis of relative gene expression data using real-time quantitative PCR and the 2(-Delta Delta C(T)) Method. Methods 25: 402-408, 2001

17. Xiao S, Yang Z, Lv R, Zhao J, Wu M, Liao Y and Liu Q: miR-135b contributes to the radioresistance by targeting GSK3 $\beta$ in human glioblastoma multiforme cells. PLoS One 9: e108810, 2014.

18. Tang R, Liang L, Luo D, Feng Z, Huang Q, He R, Gan T, Yang L and Chen G: Downregulation of miR-30a is associated with poor prognosis in lung cancer. Med Sci Monit 21: 2514-2520, 2015.

19. Müller AH, Povlsen GK, Bang-Berthelsen CH, Kruse LS, Nielsen J, Warfvinge $\mathrm{K}$ and Edvinsson L: Regulation of microRNAs miR-30a and miR-143 in cerebral vasculature after experimental subarachnoid hemorrhage in rats. BMC Genomics 16: 119, 2015.

20. Wen Y, Han J, Chen J, Dong J, Xia Y, Liu J, Jiang Y, Dai J, Lu J, Jin G, et al: Plasma miRNAs as early biomarkers for detecting hepatocellular carcinoma. Int J Cancer 137: 1679-1690, 2015.

21. Guess MG, Barthel KK, Harrison BC and Leinwand LA: miR-30 family microRNAs regulate myogenic differentiation and provide negative feedback on the microRNA pathway. PLoS One 10: e0118229, 2015.

22. Long G, Wang F, Li H, Yin Z, Sandip C, Lou Y, Wang Y, Chen C and Wang DW: Circulating miR-30a, miR-126 and let-7b as biomarker for ischemic stroke in humans. BMC Neurol 13: 178, 2013.

23. Zhang Q, Tang Q, Qin D, Yu L, Huang R, Lv G, Zou Z, Jiang XC, Zou C, Liu W, et al: Role of microRNA 30a targeting insulin receptor substrate 2 in colorectal tumorigenesis. Mol Cell Biol 35: 988-1000, 2015.

24. Wang T, Li F and Tang S: MiR-30a upregulates BCL2A1, IER3 and cyclin D2 expression by targeting FOXL2. Oncol Lett 9: 967-971, 2015.

25. Wang P, Liang J, Li Y, Li J, Yang X, Zhang X, Han S, Li S and Li J: Down-regulation of miRNA-30a alleviates cerebral ischemic injury through enhancing beclin 1-mediated autophagy. Neurochem Res 39: 1279-1291, 2014.

26. McCullough LD, Tarabishy S, Liu L, Benashski S, Xu Y, Ribar T, Means A and Li J: Inhibition of calcium/calmodulin-dependent protein kinase kinase $\beta$ and calcium/calmodulin-dependent protein kinase IV is detrimental in cerebral ischemia. Stroke 44: 2559-2566, 2013.

27. Koval AV, Vlasov P, Shichkova P, Khunderyakova S, Markov Y, Panchenko J, Volodina A, Kondrashov FA and Katanaev VL: Anti-leprosy drug clofazimine inhibits growth of triple-negative breast cancer cells via inhibition of canonical Wnt signaling. Biochem Pharmacol 87: 571-578, 2014.

28. Wang C, Hu J, Lu M, Gu H, Zhou X, Chen X, Zen K, Zhang CY, Zhang T, Ge J, et al: A panel of five serum miRNAs as a potential diagnostic tool for early-stage renal cell carcinoma. Sci Rep 5: $7610,2015$.

29. Clancy C, Joyce MR and Kerin MJ: The use of circulating microRNAs as diagnostic biomarkers in colorectal cancer. Cancer Biomark 15: 103-113, 2015.

30. Chen LT, Xu SD, Xu H, Zhang JF, Ning JF and Wang SF: MicroRNA-378 is associated with non-small cell lung cancer brain metastasis by promoting cell migration, invasion and tumor angiogenesis. Med Oncol 29: 1673-1680, 2012.

31. You L, Gu W, Chen L, Pan L, Chen J and Peng Y: MiR-378 overexpression attenuates high glucose-suppressed osteogenic differentiation through targeting CASP3 and activating PI3K/Akt signaling pathway. Int J Clin Exp Pathol 7: 7249-7261, 2014.

32. Chen Z, Li C, Xu Y, Li Y, Yang H and Rao L: Circulating level of miR-378 predicts left ventricular hypertrophy in patients with aortic stenosis. PLoS One 9: e105702, 2014.

33. Pan D, Mao C, Quattrochi B, Friedline RH, Zhu LJ, Jung DY, Kim JK, Lewis B and Wang YX: MicroRNA-378 controls classical brown fat expansion to counteract obesity. Nat Commun 5: 4725, 2014.

34. Meenhuis A, van Veelen PA, de Looper H, van Boxtel N, van den Berge IJ, Sun SM, Taskesen E, Stern P, de Ru AH, van Adrichem AJ, et al: MiR-17/20/93/106 promote hematopoietic cell expansion by targeting sequestosome 1-regulated pathways in mice. Blood 118: 916-925, 2011.

35. Fallah P, Amirizadeh N, Poopak B, Toogeh G, Arefian E, Kohram F, Hosseini Rad SM, Kohram M, Teimori Naghadeh H and Soleimani M: Expression pattern of key microRNAs in patients with newly diagnosed chronic myeloid leukemia in chronic phase. Int J Lab Hematol 37: 560-568, 2015. 
36. Shiotani A, Murao T, Kimura Y, Matsumoto H, Kamada T, Kusunoki H, Inoue K, Uedo N, Iishi H and Haruma K: Identification of serum miRNAs as novel non-invasive biomarkers for detection of high risk for early gastric cancer. Br J Cancer 109: 2323-2330, 2013

37. Mei Z, Su T, Ye J, Yang C, Zhang S and Xie C: The miR-15 family enhances the radiosensitivity of breast cancer cells by targeting G2 checkpoints. Radiat Res 183: 196-207, 2015.
38. Maudet C, Mano M, Sunkavalli U, Sharan M, Giacca M, Förstner KU and Eulalio A: Functional high-throughput screening identifies the miR-15 microRNA family as cellular restriction factors for Salmonella infection. Nat Commun 5: 4718, 2014.

39. Tijsen AJ, van der Made I, van den Hoogenhof MM, Wijnen WJ, van Deel ED, de Groot NE, Alekseev S, Fluiter K, Schroen B, Goumans MJ, et al: The microRNA-15 family inhibits the TGF $\beta$-pathway in the heart. Cardiovasc Res 104: 61-71, 2014 